УДК 533.72

\author{
V. D. Gordevskyy, O. O. Hukalov
}

\title{
THE INTERACTION OF THE ASYMMETRICAL SCREW FLOWS FOR THE BRYAN-PIDDUCK MODEL
}

\begin{abstract}
V. D. Gordevskyy, O. O. Hukalov. The interaction of the asymmetrical screw flows for the Bryan-Pidduck model, Mat. Stud. 50 (2018), 173-188.

Some approximate solutions of the Boltzmann equation for a model of rough spheres are constructed. Several sufficient conditions which minimize the uniform-integral error between parts of the Bryan-Pidduck equation are obtained.
\end{abstract}

1. Introduction. In this paper, we consider a model of rough spheres ([1]) first by introduced by Bryan in 1894 ([2]). The methods developed by Chapman and Enskog for general nonrotating spherical molecules were extended to Bryan's model by Pidduck in 1922 ([3]). The advantage of the model over all other variably rotating models is that no variables specifying its orientation in the space are required.

The statement that the molecules are perfectly elastic and perfectly rough is to be interpreted as follows. When two molecules collide, the points which come into contact will not, in general, possess the same velocity. It is supposed that the two spheres grip each other without slipping; first each sphere is strained by the other, and then the strain energy is reconverted into kinetic energy of translation and rotation, no energy being lost; the effect is that the relative velocity of the spheres at their point of contact is reversed by the impact.

The model applies to monatomic molecules and, given the possibility of rotation, is more physical than the model of hard balls and interesting to explore.

The Boltzmann equation for the model of rough spheres (or the Bryan-Pidduck equation) has the form $([1]-[6])$

$$
\begin{gathered}
D(f)=Q(f, f) \\
D(f) \equiv \frac{\partial f}{\partial t}+\left(V, \frac{\partial f}{\partial x}\right) ; \\
Q(f, f) \equiv \frac{d^{2}}{2} \int_{R^{3}} d V_{1} \int_{R^{3}} d \omega_{1} \int_{\Sigma} d \alpha B\left(V-V_{1}, \alpha\right) \times \\
\times\left[f\left(t, V_{1}^{*}, x, \omega_{1}^{*}\right) f\left(t, V^{*}, x, \omega^{*}\right)-f(t, V, x, \omega) f\left(t, V_{1}, x, \omega_{1}\right)\right] .
\end{gathered}
$$

We use the following notation: $d$ is the diameter of the molecule, which is connected with the moment of inertia $I$ by the relation

$$
I=\frac{b d^{2}}{4}
$$

2010 Mathematics Subject Classification: 76P05, 45K05, 82C40, 35Q55.

Keywords: the Bryan-Pidduck equation; bimodal distribution; uniform-integral error. doi:10.15330/ms.50.2.173-188

(C) V. D. Gordevskyy, O. O. Hukalov, 2018 
where $b\left(b \in\left(0, \frac{2}{3}\right]\right)$ is the parameter characterizing the isotropic distribution of matter inside the gas particles; $t$ is the time; $x=\left(x^{1}, x^{2}, x^{3}\right) \in R^{3}$ is the spatial coordinate; threedimensional vectors $V=\left(V^{1}, V^{2}, V^{3}\right)$ and $\omega=\left(\omega^{1}, \omega^{2}, \omega^{3}\right)$ are the linear and angular velocities of the molecule, respectively; $\frac{\partial f}{\partial x}$ is the gradient of the function $f$ of the variable $x ; \Sigma$ is the unit sphere in the space $R^{3} ; \alpha$ is the unit vector of $R^{3}$, directed along the line connecting the centers of the colliding molecules;

$$
B\left(V-V_{1}, \alpha\right)=\left|\left(V-V_{1}, \alpha\right)\right|-\left(V-V_{1}, \alpha\right)
$$

is the collision term in the collision integral (3).

The linear $\left(V^{*}, V_{1}^{*}\right)$ and angular $\left(\omega^{*}, \omega_{1}^{*}\right)$ molecular velocities after the collision can be expressed by the appropriate values before the collision by the following formulas [1]

$$
\begin{aligned}
& V^{*}=V-\frac{1}{b+1}\left(b\left(V_{1}-V\right)-\frac{b d}{2}\left[\alpha, \omega+\omega_{1}\right]+\alpha\left(\alpha, V_{1}-V\right)\right), \\
& V_{1}^{*}=V_{1}+\frac{1}{b+1}\left(b\left(V_{1}-V\right)-\frac{b d}{2}\left[\alpha, \omega+\omega_{1}\right]+\alpha\left(\alpha, V_{1}-V\right)\right), \\
& \omega^{*}=\omega+\frac{2}{d(b+1)}\left\{\left[\alpha, V-V_{1}\right]+\frac{d}{2}\left(\alpha\left(\omega+\omega_{1}, \alpha\right)-\omega-\omega_{1}\right)\right\}, \\
& \omega_{1}^{*}=\omega_{1}+\frac{2}{d(b+1)}\left\{\left[\alpha, V-V_{1}\right]+\frac{d}{2}\left(\alpha\left(\omega+\omega_{1}, \alpha\right)-\omega-\omega_{1}\right)\right\},
\end{aligned}
$$

where the symbol $[a, b]$ indicates the vector product of vectors $a$ and $b$. These formulas can be obtained using the laws of conservation of momentum and the total energy of translational and rotational motion.

2. Statement of the problem. As a value of deviation between the parts of equation (1)-(3) we use the uniform-integral error from [6]

$$
\Delta=\sup _{(t, x) \in R^{4}} \int_{R^{3}} d V \int_{R^{3}} d \omega|D(f)-Q(f, f)|,
$$

Still in the works ([7], [8]) we will seek an approximate solution of the equation (1)-(3) in the form of a bimodal distribution

$$
f=\varphi_{1} M_{1}+\varphi_{2} M_{2},
$$

where the functions $\varphi_{i}=\varphi_{i}(t, x)$ (here and then the index $i$ by default takes values 1 and 2) are non-negative and smooth. In addition they are chosen to be such that the deviation between the parts of equation (1) for function (9) is arbitrarily small.

The only exact solutions of the Boltzmann equation, which are known in the explicit form are Maxwellians $M_{i}$, moreover

$$
D\left(M_{i}\right)=0, \quad Q\left(M_{i}, M_{i}\right)=0 .
$$

In this paper, we will consider one of the possible types of local Maxwellians [9], namely those that describe the screw movement. They have the following form [9]

$$
M_{i}=\rho_{0 i} e^{\beta_{i} \bar{\omega}_{i}^{2} r_{i}^{2}} I^{3 / 2}\left(\frac{\beta_{i}}{\pi}\right)^{3} e^{-\beta_{i}\left(\left(V-\bar{V}_{i}\right)^{2}+I \omega^{2}\right)},
$$


$\rho_{0 i}$ is an arbitrary positive constant, $\beta_{i}=\frac{1}{2 T_{i}}$ is a value inverse to the absolute temperature $T_{i}$, $\bar{\omega}_{i}$ is a constant angular flow rate. The mass velocity of molecules $\bar{V}_{i}$ has the form

$$
\bar{V}_{i}=\widehat{V}_{i}+\left[\bar{\omega}_{i}, x\right]
$$

$\widehat{V}_{i}$ is an arbitrary three-dimensional vector. By $r_{i}$ indicated the distance to the axis of rotation $x_{0 i}$

$$
r_{i}^{2}=\frac{1}{\bar{\omega}_{i}^{2}}\left[\bar{\omega}_{i}, x-x_{0 i}\right]^{2}, \quad x_{0 i}=\frac{\left[\bar{\omega}_{i}, \widehat{V}_{i}\right]}{\bar{\omega}_{i}^{2}} .
$$

It should be noted that the product $\rho_{0 i} e^{\beta_{i} \bar{\omega}_{i}^{2} r_{i}^{2}}$ is also called the density $\rho_{i}$ of a gas.

We note that the Maxwellian which we will consider is a partial case of propellant currents with a vortex structure and have a rather simple form[9]. These functions do not have any features.

We are searching an approximate solution of the Boltzmann equation, which means the following. We need to find these features $\varphi_{i}$ iз (9), to the value of the error $\Delta$ it was possible to make it arbitrary small due to the selection of conditions for hydrodynamic flow parameters.

For convenience of further calculations, we write the unknown coefficient functions $\varphi_{i}$ in the following form

$$
\varphi_{i}(t, x)=\psi_{i}(t, x) e^{-\beta_{i} \bar{\omega}_{i}^{2} r_{i}^{2}}
$$

where to the new functions $\psi_{i}$ the same conditions of non-negative and smoothness on $R^{4}$ are supposed as earlier.

In addition, we assume that the angular velocity of the gas flow will be expressed as follows

$$
\bar{\omega}_{i}=\bar{\omega}_{0 i} q_{i} \beta_{i}^{-m_{i}}
$$

$\bar{\omega}_{0 i}$ is an arbitrary vector with $R^{3}, q_{i}>0$ is positive constant. If $\beta_{i}$ will go to infinity, then the angular velocity tends to the zero vector, which from the physical point of view means the slowing of the rotation at low-temperature regime.

In previous works([5]-[8]), devoted to the search for approximate solutions of the Boltzmann equation for a model of rough spheres, the flows considered had the same degree of slowing of angular velocity. In this paper, for the first time, the behavior of an approximate solution is studied, when the flows will have different degrees. A similar problem was considered for a simpler physical meaning of the hard sphere model in the article [10].

3. The main results. In the first theorem, we assume that the angular velocity of the gas for different flows, with the same degree of velocity, goes to a zero vector, that is, $m_{1}=m_{2}$.

Theorem 1. Let the conditions be fulfilled (14), (15) and the following functions

$$
\psi_{i},|x| \psi_{i},\left|\frac{\partial \psi_{i}}{\partial t}\right|,\left|\frac{\partial \psi_{i}}{\partial x}\right|,|x|\left|\frac{\partial \psi_{i}}{\partial x}\right|
$$

are bounded throughout the space $(t, x) \in R^{4}$.

Then there exists a value $\Delta^{\prime}$, such that

$$
\Delta \leqslant \Delta^{\prime},
$$


and depending on the indicators $m_{i}$ there is a low temperature limit of $\Delta^{\prime}$, which has the following form there

a) if $m_{i}>\frac{1}{2}$ or $\frac{1}{4}<m_{i}<\frac{1}{2}$ with an additional condition $\bar{\omega}_{0 i} \| \widehat{V}_{i}$ then the next limit is

$$
\boldsymbol{Y}:=\lim _{\beta_{i} \rightarrow+\infty} \Delta^{\prime}=\sum_{i=1}^{2} \rho_{0 i} \sup _{(t, x) \in R^{4}}\left|\frac{\partial \psi_{i}}{\partial t}+\left(\widehat{V}_{i}, \frac{\partial \psi_{i}}{\partial x}\right)\right|+4 \pi d^{2} \rho_{01} \rho_{02}\left|\widehat{V}_{1}-\widehat{V}_{2}\right| \sup _{(t, x) \in R^{4}} \psi_{1} \psi_{2} ;
$$

b) when $m_{i}=\frac{1}{2}$, then the statement is valid

$$
\lim _{\beta_{i} \rightarrow+\infty} \Delta^{\prime}=\boldsymbol{Y}+\frac{4}{\sqrt{\pi}} \sum_{i=1}^{2} \rho_{0 i} q_{i}\left|\left[\bar{\omega}_{0 i}, \widehat{V}_{i}\right]\right| \sup _{(t, x) \in R^{4}} \psi_{i}
$$

c) if $m_{i}=\frac{1}{4}$ and $\bar{\omega}_{0 i} \| \widehat{V}_{i}$ we have

$$
\lim _{\beta_{i} \rightarrow+\infty} \Delta^{\prime}=\boldsymbol{Y}+\frac{4}{\sqrt{\pi}} \sum_{i=1}^{2} \rho_{0 i}\left|\bar{\omega}_{0 i}\right|^{2} q_{i}^{2} \sup _{(t, x) \in R^{4}}|x| \psi_{i}
$$

Proof. First, we will make a substitution of the bimodal distribution (9) in the differential operator $D(f)$

$$
\begin{gathered}
D(f)=\frac{\partial}{\partial t}\left(\varphi_{1} M_{1}+\varphi_{2} M_{2}\right)+\left(V, \frac{\partial}{\partial x}\left(\varphi_{1} M_{1}+\varphi_{2} M_{2}\right)\right)= \\
=\varphi_{1} \frac{\partial M_{1}}{\partial t}+M_{1} \frac{\partial \varphi_{1}}{\partial t}+\varphi_{2} \frac{\partial M_{2}}{\partial t}+M_{2} \frac{\partial \varphi_{2}}{\partial t}+\left(V, \varphi_{1} \frac{\partial M_{1}}{\partial x}+M_{1} \frac{\partial \varphi_{1}}{\partial x}+\varphi_{2} \frac{\partial M_{2}}{\partial x}+M_{2} \frac{\partial \varphi_{2}}{\partial x}\right)= \\
=\varphi_{1} D\left(M_{1}\right)+\varphi_{2} D\left(M_{2}\right)+M_{1} D\left(\varphi_{1}\right)+M_{2} D\left(\varphi_{2}\right),
\end{gathered}
$$

and because the Maxwellians $M_{i}$ are exact solutions of the equation (1)-(3), we will have

$$
D(f)=M_{1} D\left(\varphi_{1}\right)+M_{2} D\left(\varphi_{2}\right) .
$$

Then we will find the form of collision integral $Q(f, f)$

$$
\begin{gathered}
Q(f, f)=\frac{d^{2}}{2} \int_{R^{3}} d V_{1} \int_{R^{3}} d \omega_{1} \int_{\Sigma} d \alpha B\left(V-V_{1}, \alpha\right) \times \\
\times\left[\left(\varphi_{1} M_{1}\left(V_{1}^{*}, \omega_{1}^{*}\right)+\varphi_{2} M_{2}\left(V_{1}^{*}, \omega_{1}^{*}\right)\right)\left(\varphi_{1} M_{1}\left(V^{*}, \omega^{*}\right)+\varphi_{2} M_{2}\left(V^{*}, \omega^{*}\right)\right)-\right. \\
\left.-\left(\varphi_{1} M_{1}\left(V_{1}, \omega_{1}\right)+\varphi_{2} M_{2}\left(V_{1}, \omega_{1}\right)\right)\left(\varphi_{1} M_{1}(V, \omega)+\varphi_{2} M_{2}(V, \omega)\right)\right],
\end{gathered}
$$

opening the brackets in the subintegral expression and, performing the rearrangement of the terms, we have

$$
Q(f, f)=\varphi_{1}^{2} Q\left(M_{1}, M_{1}\right)+\varphi_{2}^{2} Q\left(M_{2}, M_{2}\right)+\varphi_{1} \varphi_{2} Q\left(M_{1}, M_{2}\right)+\varphi_{1} \varphi_{2} Q\left(M_{2}, M_{1}\right) .
$$

Since the first two terms vanish thanks to (10), finally we have

$$
Q(f, f)=\varphi_{1} \varphi_{2}\left(Q\left(M_{1}, M_{2}\right)+Q\left(M_{2}, M_{1}\right)\right)
$$


As you know [11], collision integral can be written as follows

$$
\begin{gathered}
Q(f, g)=G(f, g)-f L(g), \\
G(f, g)=\frac{d^{2}}{2} \int_{R^{3}} d V_{1} \int_{R^{3}} d \omega_{1} \int_{\sum^{2}} d \alpha B\left(V-V_{1}, \alpha\right) f\left(t, x, V_{1}^{*}, \omega_{1}^{*}\right) g\left(t, x, V^{*}, \omega^{*}\right), \\
L(g)=\frac{d^{2}}{2} \int_{R^{3}} d V_{1} \int_{R^{3}} d \omega_{1} \int_{\sum} d \alpha B\left(V-V_{1}, \alpha\right) g\left(t, x, V_{1}, \omega_{1}\right),
\end{gathered}
$$

the function $G(f, g)$ is the gain term, and $L(g)$ is the loss term of the collision integral.

In the paper [8] was shown that

$$
\int_{R^{3}} d V \int_{R^{3}} d \omega Q\left(M_{i}, M_{j}\right)=0
$$

from what, thanks (23) follows the following equality

$$
\int_{R^{3}} d V \int_{R^{3}} d \omega G\left(M_{i}, M_{j}\right)=\int_{R^{3}} d V \int_{R^{3}} d \omega M_{i} L\left(M_{j}\right) .
$$

Now using the expressions (21), (22) for function (9) we will find an estimate for the under integral expression in the error (8), applying (23)

$$
\begin{gathered}
|D(f)-Q(f, f)|=\left|M_{1} D\left(\varphi_{1}\right)+M_{2} D\left(\varphi_{2}\right)-\varphi_{1} \varphi_{2}\left(Q\left(M_{1}, M_{2}\right)+Q\left(M_{2}, M_{1}\right)\right)\right|= \\
=\left|M_{1} D\left(\varphi_{1}\right)+M_{2} D\left(\varphi_{2}\right)-\varphi_{1} \varphi_{2}\left\{G\left(M_{1}, M_{2}\right)-M_{1} L\left(M_{2}\right)+G\left(M_{2}, M_{1}\right)-M_{2} L\left(M_{1}\right)\right\}\right| \leqslant \\
\leqslant M_{1}\left|D\left(\varphi_{1}\right)+\varphi_{1} \varphi_{2} L\left(M_{2}\right)\right|+M_{2}\left|D\left(\varphi_{2}\right)+\varphi_{1} \varphi_{2} L\left(M_{1}\right)\right|+\varphi_{1} \varphi_{2}\left(G\left(M_{1}, M_{2}\right)+G\left(M_{2}, M_{1}\right)\right) .
\end{gathered}
$$

Let us integrate the last inequality over the whole space of linear and angular velocities

$$
\begin{gathered}
\int_{R^{3}} d V \int_{R^{3}} d \omega|D(f)-Q(f, f)| \leqslant \sum_{\substack{i, j=1 \\
i \neq j}}^{2} \int_{R^{3}} d V \int_{R^{3}} d \omega\left|D\left(\varphi_{i}\right)+\varphi_{i} \varphi_{j} L\left(M_{j}\right)\right| M_{i}+ \\
+\varphi_{1} \varphi_{2} \int_{R^{3}} d V \int_{R^{3}} d \omega\left(G\left(M_{1}, M_{2}\right)+G\left(M_{2}, M_{1}\right)\right) \leqslant \\
\leqslant \sum_{i=1}^{2} \int_{R^{3}} d V \int_{R^{3}} d \omega\left|D\left(\varphi_{i}\right)\right| M_{i}+\sum_{\substack{i, j=1 \\
i \neq j}}^{2} \varphi_{i} \varphi_{j} \int_{R^{3}} d V \int_{R^{3}} d \omega M_{i} L\left(M_{j}\right)+ \\
+2 \varphi_{1} \varphi_{2} \int_{R^{3}} d V \int_{R^{3}} d \omega G\left(M_{1}, M_{2}\right)
\end{gathered}
$$

and taking into account equality(27) and non-negativity of the integral expression for $G\left(M_{1}, M_{2}\right)(24)$, we get

$$
\begin{gathered}
\int_{R^{3}} d V \int_{R^{3}} d \omega|D(f)-Q(f, f)| \leqslant \\
\leqslant \sum_{i=1}^{2} \int_{R^{3}} d V \int_{R^{3}} d \omega\left|D\left(\varphi_{i}\right)\right| M_{i}+4 \varphi_{1} \varphi_{2} \int_{R^{3}} d V \int_{R^{3}} d \omega G\left(M_{1}, M_{2}\right) .
\end{gathered}
$$


To find $D\left(\varphi_{i}\right)$, let us calculate derivative of the functions $\varphi_{i}$ using the expression (14). The derivative on time has a simple form

$$
\frac{\partial \varphi_{i}}{\partial t}=e^{-\beta_{i} \bar{\omega}_{i}^{2} r_{i}^{2}} \frac{\partial \psi_{i}}{\partial t}
$$

but on the spatial coordinate $x$

$$
\begin{gathered}
\frac{\partial \varphi_{i}}{\partial x}=e^{-\beta_{i} \bar{\omega}_{i}^{2} r_{i}^{2}} \frac{\partial \psi_{i}}{\partial x}+\psi_{i} e^{-\beta_{i} \bar{\omega}_{i}^{2} r_{i}^{2}} \frac{\partial}{\partial x}\left(-\beta_{i} \bar{\omega}_{i}^{2} r_{i}^{2}\right)= \\
=\frac{\partial \psi_{i}}{\partial x}+\psi_{i} e^{-\beta_{i} \bar{\omega}_{i}^{2} r_{i}^{2}}\left(-\beta_{i}\right) \cdot 2\left[\left[\bar{\omega}_{i}, x-x_{0 i}\right], \bar{\omega}_{i}\right]=e^{-\beta_{i} \bar{\omega}_{i}^{2} r_{i}^{2}}\left(\frac{\partial \psi_{i}}{\partial x}+2 \psi_{i} \beta_{i}\left[\bar{\omega}_{i},\left[\bar{\omega}_{i}, x-x_{0 i}\right]\right]\right)= \\
=e^{-\beta_{i} \bar{\omega}_{i}^{2} r_{i}^{2}}\left(\frac{\partial \psi_{i}}{\partial x}+2 \bar{\omega}_{i} \psi_{i} \beta_{i}\left(\bar{\omega}_{i}, x-x_{0 i}\right)-2 \beta_{i} \psi_{i} \bar{\omega}_{i}^{2}\left(x-x_{0 i}\right)\right),
\end{gathered}
$$

and, taking into account that $x_{0 i} \perp \bar{\omega}_{i}$ (13), we obtain the spatial gradient of the coefficient function $\varphi_{i}(14)$

$$
\frac{\partial \varphi_{i}}{\partial x}=e^{-\beta_{i} \bar{\omega}_{i}^{2} r_{i}^{2}}\left(\frac{\partial \psi_{i}}{\partial x}+2 \bar{\omega}_{i} \psi_{i} \beta_{i}\left(\bar{\omega}_{i}, x\right)-2 \beta_{i} \psi_{i} \bar{\omega}_{i}^{2}\left(x-x_{0 i}\right)\right) .
$$

We make a substitution of Maxwellians $M_{i}$ and derivatives (29), (30) into the previous inequality (28), while reducing the exponent

$$
\begin{gathered}
\int_{R^{3}} d V \int_{R^{3}} d \omega|D(f)-Q(f, f)| \leqslant I^{3 / 2} \pi^{-3} \sum_{i=1}^{2} \int_{R^{3}} d V \int_{R^{3}} d \omega \rho_{0 i} \beta_{i}^{3} e^{-\beta_{i}\left(\left(V-\bar{V}_{i}\right)^{2}+I \omega^{2}\right)} \times \\
\times\left|\frac{\partial \psi_{i}}{\partial t}+\left(V, \frac{\partial \psi_{i}}{\partial x}\right)+2 \beta_{i} \psi_{i}\left(V, \bar{\omega}_{i}\right)\left(\bar{\omega}_{i}, x\right)-2 \beta_{i} \psi_{i} \bar{\omega}_{i}^{2}\left(V, x-x_{0 i}\right)\right|+ \\
+4 \varphi_{1} \varphi_{2} \int_{R^{3}} d V \int_{R^{3}} d \omega G\left(M_{1}, M_{2}\right) .
\end{gathered}
$$

For further transformations, we will need the equality that was obtained in the work [6]

$$
\int_{R^{3}} d V \int_{R^{3}} d \omega G\left(M_{1}, M_{2}\right)=\frac{d^{2} \rho_{1} \rho_{2}}{\pi^{2}} \int_{R^{3}} \int_{R^{3}} d u d u_{1} e^{-u^{2}-u_{1}^{2}}\left|\frac{u}{\sqrt{\beta_{1}}}+\bar{V}_{1}-\frac{u_{1}}{\sqrt{\beta_{2}}}-\bar{V}_{2}\right| .
$$

Using the last equality and integrating with respect to the space of the angular velocity $\omega$ we obtain

$$
\begin{gathered}
\int_{R^{3}} d V \int_{R^{3}} d \omega|D(f)-Q(f, f)| \leqslant \pi^{-3 / 2} \sum_{i=1}^{2} \int_{R^{3}} d V \rho_{0 i} \beta_{i}^{3 / 2} e^{-\beta_{i}\left(V-\bar{V}_{i}\right)^{2}} \times \\
\times\left|\frac{\partial \psi_{i}}{\partial t}+\left(V, \frac{\partial \psi_{i}}{\partial x}\right)+2 \beta_{i} \psi_{i}\left(V, \bar{\omega}_{i}\right)\left(\bar{\omega}_{i}, x\right)-2 \beta_{i} \psi_{i} \bar{\omega}_{i}^{2}\left(V, x-x_{0 i}\right)\right|+ \\
\quad+\frac{4 d^{2} \rho_{01} \rho_{02} \psi_{1} \psi_{2}}{\pi^{2}} \int_{R^{3}} \int_{R^{3}} d u d u_{1} e^{-u^{2}-u_{1}^{2}}\left|\frac{u}{\sqrt{\beta_{1}}}+\bar{V}_{1}-\frac{u_{1}}{\sqrt{\beta_{2}}}-\bar{V}_{2}\right| .
\end{gathered}
$$

In the integral under the sum we will make a replacement

$$
p=\left(V-\bar{V}_{i}\right) \sqrt{\beta_{i}},
$$


and, taking into account the Jacobian $J=\frac{1}{\beta_{i}^{3 / 2}}$ we have

$$
\begin{gathered}
\int_{R^{3}} d V \int_{R^{3}} d \omega|D(f)-Q(f, f)| \leqslant \pi^{-3 / 2} \sum_{i=1}^{2} \int_{R^{3}} d p \rho_{0 i} e^{-p^{2}} \mid \frac{\partial \psi_{i}}{\partial t}+\left(\frac{p}{\sqrt{\beta_{i}}}+\bar{V}_{i}, \frac{\partial \psi_{i}}{\partial x}\right)+ \\
+2 \beta_{i} \psi_{i}\left(\frac{p}{\sqrt{\beta_{i}}}+\bar{V}_{i}, \bar{\omega}_{i}\right)\left(\bar{\omega}_{i}, x\right)-2 \beta_{i} \psi_{i} \bar{\omega}_{i}^{2}\left(\frac{p}{\sqrt{\beta_{i}}}+\bar{V}_{i}, x-x_{0 i}\right) \mid+ \\
+\frac{4 d^{2} \rho_{01} \rho_{02} \psi_{1} \psi_{2}}{\pi^{2}} \int_{R^{3}} \int_{R^{3}} d u d u_{1} e^{-u^{2}-u_{1}^{2}}\left|\bar{V}_{1}-\bar{V}_{2}+\frac{u}{\sqrt{\beta_{1}}}-\frac{u_{1}}{\sqrt{\beta_{2}}}\right|
\end{gathered}
$$

Taking into account (12) and using the properties of the scalar product, we will have

$$
\begin{gathered}
\int_{R^{3}} d V \int_{R^{3}} d \omega|D(f)-Q(f, f)| \leqslant \\
\leqslant \pi^{-3 / 2} \sum_{i=1}^{2} \int_{R^{3}} d p \rho_{0 i} e^{-p^{2}} \mid \frac{\partial \psi_{i}}{\partial t}+\left(\frac{p}{\sqrt{\beta_{i}}}+\widehat{V}_{i}+\left[\bar{\omega}_{i}, x\right], \frac{\partial \psi_{i}}{\partial x}\right)+ \\
+2 \beta_{i} \psi_{i}\left(\frac{p}{\sqrt{\beta_{i}}}+\widehat{V}_{i}, \bar{\omega}_{i}\right)\left(\bar{\omega}_{i}, x\right)-2 \beta_{i} \psi_{i} \bar{\omega}_{i}^{2}\left(\frac{p}{\sqrt{\beta_{i}}}+\widehat{V}_{i}+\left[\bar{\omega}_{i}, x\right], x-\frac{1}{\bar{\omega}_{i}^{2}}\left[\bar{\omega}_{i}, \widehat{V}_{i}\right]\right) \mid+ \\
+\frac{4 d^{2} \rho_{01} \rho_{02} \psi_{1} \psi_{2}}{\pi^{2}} \times \int_{R^{3}} \int_{R^{3}} d u d u_{1} e^{-u^{2}-u_{1}^{2}}\left|\widehat{V}_{1}-\widehat{V}_{2}+\frac{u}{\sqrt{\beta_{1}}}-\frac{u_{1}}{\sqrt{\beta_{2}}}-\left[\bar{\omega}_{1}-\bar{\omega}_{2}, x\right]\right| .
\end{gathered}
$$

With the help of the following formula of the vector algebra

$$
\forall a, b, c, d \in R^{3}: \quad([a, b],[c, d])=(a, c)(b, d)-(a, d)(b, c)
$$

we get that

$$
\left(\frac{p}{\sqrt{\beta_{i}}}+\widehat{V}_{i}+\left[\bar{\omega}_{i}, x\right], x-\frac{1}{\bar{\omega}_{i}^{2}}\left[\bar{\omega}_{i}, \widehat{V}_{i}\right]\right)=\left(\frac{p}{\sqrt{\beta_{i}}}, x-\frac{1}{\bar{\omega}_{i}^{2}}\left[\bar{\omega}_{i}, \widehat{V}_{i}\right]\right)+\frac{1}{\bar{\omega}_{i}^{2}}\left(\bar{\omega}_{i}, \widehat{V}_{i}\right)\left(\bar{\omega}_{i}, x\right) .
$$

Thanks to the last equality, we can continue to evaluate (33)

$$
\begin{gathered}
\int_{R^{3}} d V \int_{R^{3}} d \omega|D(f)-Q(f, f)| \leqslant \\
\leqslant \pi^{-3 / 2} \sum_{i=1}^{2} \int_{R^{3}} d p \rho_{0 i} e^{-p^{2}} \mid \frac{\partial \psi_{i}}{\partial t}+\left(\frac{p}{\sqrt{\beta_{i}}}+\widehat{V}_{i}+\left[\bar{\omega}_{i}, x\right], \frac{\partial \psi_{i}}{\partial x}\right)+ \\
+2 \beta_{i} \psi_{i}\left(\frac{p}{\sqrt{\beta_{i}}}, \bar{\omega}_{i}\right)\left(\bar{\omega}_{i}, x\right)-2 \sqrt{\beta_{i}} \psi_{i} \bar{\omega}_{i}^{2}(p, x)+2 \beta_{i} \psi_{i}\left(\frac{p}{\sqrt{\beta_{i}}},\left[\bar{\omega}_{i}, \widehat{V}_{i}\right]\right) \mid+ \\
+\frac{4 d^{2} \rho_{01} \rho_{02} \psi_{1} \psi_{2}}{\pi^{2}} \int_{R^{3}} \int_{R^{3}} d u d u_{1} e^{-u^{2}-u_{1}^{2}}\left|\widehat{V}_{1}-\widehat{V}_{2}+\frac{u}{\sqrt{\beta_{1}}}-\frac{u_{1}}{\sqrt{\beta_{2}}}-\left[\bar{\omega}_{1}-\bar{\omega}_{2}, x\right]\right| .
\end{gathered}
$$


After a few of elementary transformations, we finally have

$$
\begin{gathered}
\int_{R^{3}} d V \int_{R^{3}} d \omega|D(f)-Q(f, f)| \leq \\
\leqslant \pi^{-3 / 2} \sum_{i=1}^{2} \int_{R^{3}} d p \rho_{0 i} e^{-p^{2}} \mid \frac{\partial \psi_{i}}{\partial t}+\left(\frac{p}{\sqrt{\beta_{i}}}+\widehat{V}_{i}+\left[\bar{\omega}_{i}, x\right], \frac{\partial \psi_{i}}{\partial x}\right)+ \\
+2 \sqrt{\beta_{i}} \psi_{i}\left\{\left(p,\left[\bar{\omega}_{i}, \widehat{V}_{i}\right]\right)-\left(\left[\bar{\omega}_{i}, p\right],\left[\bar{\omega}_{i}, x\right]\right)\right\} \mid+ \\
+\frac{4 d^{2} \rho_{01} \rho_{02} \psi_{1} \psi_{2}}{\pi^{2}} \int_{R^{3}} \int_{R^{3}} d u d u_{1} e^{-u^{2}-u_{1}^{2}}\left|\widehat{V}_{1}-\widehat{V}_{2}+\frac{u}{\sqrt{\beta_{1}}}-\frac{u_{1}}{\sqrt{\beta_{2}}}-\left[\bar{\omega}_{1}-\bar{\omega}_{2}, x\right]\right| .
\end{gathered}
$$

Supremum by the variables $t$ and $x$ of the right-hand side of the last inequality exists due to the boundness of the functions specified in the condition (16). Then, performing the transition to the supremum in the inequality (35), we receive

$$
\begin{gathered}
\Delta=\sup _{(t, x) \in R^{4}} \int_{R^{3}} d V \int_{R^{3}} d \omega|D(f)-Q(f, f)| \leqslant \\
\leqslant \pi^{-3 / 2} \sum_{i=1}^{2} \int_{R^{3}} d p \rho_{0 i} e^{-p^{2}} \sup _{(t, x) \in R^{4}} \mid \frac{\partial \psi_{i}}{\partial t}+\left(\frac{p}{\sqrt{\beta_{i}}}+\widehat{V}_{i}+\left[\bar{\omega}_{i}, x\right], \frac{\partial \psi_{i}}{\partial x}\right)+ \\
+2 \sqrt{\beta_{i}} \psi_{i}\left\{\left(p,\left[\bar{\omega}_{i}, \widehat{V}_{i}\right]\right)-\left(\left[\bar{\omega}_{i}, p\right],\left[\bar{\omega}_{i}, x\right]\right)\right\} \mid+ \\
+\frac{4 d^{2} \rho_{01} \rho_{02}}{\pi^{2}} \sup _{(t, x) \in R^{4}} \psi_{1} \psi_{2} \int_{R^{3}} \int_{R^{3}} d u d u_{1} e^{-u^{2}-u_{1}^{2}}\left|\widehat{V}_{1}-\widehat{V}_{2}+\frac{u}{\sqrt{\beta_{1}}}-\frac{u_{1}}{\sqrt{\beta_{2}}}-\left[\bar{\omega}_{1}-\bar{\omega}_{2}, x\right]\right| .
\end{gathered}
$$

We will make the redefinition $\delta_{i}:=\frac{1}{\beta_{i}}$ and take into account the decomposition (15), then according to (17) we have the expression for $\Delta^{\prime}$

$$
\begin{aligned}
\Delta^{\prime}=\pi^{-3 / 2} \sum_{i=1}^{2} \int_{R^{3}} d p \rho_{0 i} e^{-p^{2}} \sup _{(t, x) \in R^{4}} \mid \frac{\partial \psi_{i}}{\partial t}+\left(p \sqrt{\delta_{i}}+\widehat{V}_{i}+\left[\bar{\omega}_{0 i}, x\right] q_{i} \delta_{i}^{m_{i}}, \frac{\partial \psi_{i}}{\partial x}\right)+ \\
+2 \psi_{i}\left\{\left(p,\left[\bar{\omega}_{0 i}, \widehat{V}_{i}\right]\right) q_{i} \delta_{i}^{m_{i}-\frac{1}{2}}-\left(\left[\bar{\omega}_{0 i}, p\right],\left[\bar{\omega}_{0 i}, x\right]\right) q_{i}^{2} \delta_{i}^{2 m_{i}-\frac{1}{2}}\right\} \mid+ \\
\quad+\frac{4 d^{2} \rho_{01} \rho_{02}}{\pi^{2}} \sup _{(t, x) \in R^{4}} \psi_{1} \psi_{2} \int_{R^{3}} \int_{R^{3}} d u d u_{1} e^{-u^{2}-u_{1}^{2}} \times \\
\times\left|\widehat{V}_{1}-\widehat{V}_{2}+u \sqrt{\delta_{1}}-u_{1} \sqrt{\delta_{2}}-\left[\bar{\omega}_{01} q_{1} \delta_{1}^{m_{1}}-\bar{\omega}_{02} q_{2} \delta_{2}^{m_{2}}, x\right]\right| .
\end{aligned}
$$

Note that the right-hand side of (37) is continuous for the variable $\delta_{i}$, when the values $m_{i}>\frac{1}{2}$ or $\frac{1}{4}<m_{i}<\frac{1}{2}$, but need to be imposed additionally the condition $\bar{\omega}_{0 i} \| \widehat{V}_{i}$. It remains to be done the limit transition in the last equality when $\beta_{i} \rightarrow+\infty$, that is equivalent to $\delta_{i} \rightarrow+0$. The legality of such a transition it is argued by the lemma of [6] and the well-known theorem of continuity of integral, which depends on the parameter. So, we get

$$
\begin{aligned}
\lim _{\beta_{i} \rightarrow+\infty} \Delta^{\prime} & =\lim _{\delta_{i} \rightarrow+0} \Delta^{\prime}=\pi^{-3 / 2} \sum_{i=1}^{2} \int_{R^{3}} d p \rho_{0 i} e^{-p^{2}} \sup _{(t, x) \in R^{4}}\left|\frac{\partial \psi_{i}}{\partial t}+\left(\widehat{V}_{i}, \frac{\partial \psi_{i}}{\partial x}\right)\right|+ \\
& +\frac{4 d^{2} \rho_{01} \rho_{02}}{\pi^{2}} \sup _{(t, x) \in R^{4}} \psi_{1} \psi_{2} \int_{R^{3}} \int_{R^{3}} d u d u_{1} e^{-u^{2}-u_{1}^{2}}\left|\widehat{V}_{1}-\widehat{V}_{2}\right|
\end{aligned}
$$


When calculating the remaining integrals, we make sure that the statement (18) is true.

In case when $m_{i}=\frac{1}{2}$, after the limiting passage, in the first term, under the sign of the supremum the result is $2 \psi_{i}\left(p,\left[\bar{\omega}_{0 i}, \widehat{V}_{i}\right]\right) q_{i}$, and performing estimations and applying the obvious equality

$$
\int_{R^{3}}|p| e^{-p^{2}} d p=2 \pi
$$

we get (19).

Considering the last case $m_{i}=\frac{1}{4}$ and using the condition $\bar{\omega}_{0 i} \| \widehat{V}_{i}$ we will again have an additional expression under the sign of the first supremum in (38), namely

$$
2 \psi_{i}\left(\left[\bar{\omega}_{0 i}, p\right],\left[\bar{\omega}_{0 i}, x\right]\right) q_{i}^{2}
$$

and performing elementary transformations and calculating the integrals we have (20).

Let us formulate some sufficient conditions to minimize the deviation (8) in the case of validity of the statement of Theorem 1 .

Corollary 1. Let functions $\psi_{i}$ have the form

$$
\psi_{i}(t, x)=C_{i}\left(x-\widehat{V}_{i} t\right),
$$

where $C_{i}$ are non-negative, smooth functions on $R^{4}$. Additionally, suppose that one of the following conditions is true

$$
\begin{gathered}
\widehat{V}_{1}=\widehat{V}_{2} \\
\operatorname{supp} \psi_{1} \cap \operatorname{supp} \psi_{2}=\varnothing, \\
d \rightarrow 0,
\end{gathered}
$$

then in the case (18) the deviation (8) can be made arbitrary small. In cases of (19) and (20) for an infinitesimality of $\Delta$ additionally we have to demand that

$$
q_{i} \rightarrow 0 \text {. }
$$

Functions $\psi_{i}$ of the form (40) make be equal to zero the first term in (18), and validity of any of the conditions (41), (42), or (43) vanishes the second term, i.e. the value $\Delta^{\prime}$ tends to zero, but due to the non-negativity of the deviation (8) and the inequality (17), the value $\Delta$ can really be made arbitrarily small Additional terms in (19) and (20) are vanish due to the fact that $q_{i}$ tends to zero (44).

Corollary 2. Let any conditions (41), (42), (43) and (44) be fulfilled again, but functions $\psi_{i}$ will have the following form

$$
\psi_{i}(t, x)=E_{i}\left(\left[x, \widehat{V}_{i}\right]\right),
$$

where $E_{i}$ are nonnegative, smooth on $R^{4}$ functions Then the statement of corollary 1 remains valid.

The difference with the result of 1 is only in the first term (18). Since the function $\psi_{i}$ (45) does not depend on time, then the derivative on $t$ is identically equal to zero, and on the variable $x$ is equal to $\left[\widehat{V}_{i}, E_{i}^{\prime}\right]$, that is, perpendicular to the vector $\widehat{V}_{i}$, which completely subtracts the first term.

In the following theorems, in contrast to Theorem 1, we consider cases where the angular velocity of the rotations of the flows $\bar{\omega}_{i}$ with varying degrees of speed, tends to zero, that is, cases of so-called asymmetric flows. 
Theorem 2. Let the conditions of Theorem 1 be satisfied, namely (14), (15) and the functions given in(16) are bounded. Assume that the degree

$$
m_{1}=\frac{1}{2}
$$

but for the second flow $m_{2}$ is any value from the interval $\left(\frac{1}{2} ;+\infty\right)$, (let us mark it as $\left.m_{2}^{0}\right)$.

Then, as before, there exists a value $\Delta^{\prime}$, inequality (17) is valid, but its low-temperature limit is

$$
\lim _{\beta_{i} \rightarrow+\infty} \Delta^{\prime}=\boldsymbol{Y}+\frac{4 q_{1} \rho_{01}}{\sqrt{\pi}}\left|\left[\bar{\omega}_{01}, \widehat{V}_{1}\right]\right| \sup _{(t, x) \in R^{4}} \psi_{1}
$$

Proof. Using the expression for value $\Delta^{\prime}(37)$, let's give its form, taking into account the current values $m_{i}$

$$
\begin{aligned}
\Delta^{\prime}=\pi^{-3 / 2} \int_{R^{3}} d p \rho_{01} e^{-p^{2}} \sup _{(t, x) \in R^{4}} \mid \frac{\partial \psi_{1}}{\partial t}+\left(p \sqrt{\delta_{1}}+\widehat{V}_{1}+\left[\bar{\omega}_{01}, x\right] q_{1} \sqrt{\delta_{1}}, \frac{\partial \psi_{1}}{\partial x}\right)+ \\
+2 \psi_{1}\left\{\left(p,\left[\bar{\omega}_{01}, \widehat{V}_{1}\right]\right) q_{1}-\left(\left[\bar{\omega}_{01}, p\right],\left[\bar{\omega}_{01}, x\right]\right) q_{1}^{2} \sqrt{\delta_{1}}\right\} \mid+ \\
+\pi^{-3 / 2} \int_{R^{3}} d p \rho_{02} e^{-p^{2}} \sup _{(t, x) \in R^{4}} \mid \frac{\partial \psi_{2}}{\partial t}+\left(p \sqrt{\delta_{2}}+\widehat{V}_{2}+\left[\bar{\omega}_{02}, x\right] q_{2} \delta_{i}^{m_{2}^{0}}, \frac{\partial \psi_{2}}{\partial x}\right)+ \\
+2 \psi_{2}\left\{\left(p,\left[\bar{\omega}_{02}, \widehat{V}_{2}\right]\right) q_{2} \delta_{2}^{m_{2}^{0}-\frac{1}{2}}-\left(\left[\bar{\omega}_{02}, p\right],\left[\bar{\omega}_{02}, x\right]\right) q_{2}^{2} \delta_{2}^{2 m_{2}^{0}-\frac{1}{2}}\right\} \mid+ \\
\quad+\frac{4 d^{2} \rho_{01} \rho_{02}}{\pi^{2}} \sup _{(t, x) \in R^{4}} \psi_{1} \psi_{2} \int_{R^{3}} \int_{R^{3}} d u d u_{1} e^{-u^{2}-u_{1}^{2}} \times \\
\times\left|\widehat{V}_{1}-\widehat{V}_{2}+u \sqrt{\delta_{1}}-u_{1} \sqrt{\delta_{2}}-\left[\bar{\omega}_{01} q_{1} \sqrt{\delta_{1}}-\bar{\omega}_{02} q_{2} \delta_{2}^{m_{2}^{0}}, x\right]\right|
\end{aligned}
$$

Next we find the low temperature limit of $\Delta^{\prime}$, which corresponds to the limiting passage when $\delta_{i} \rightarrow+0$, taking into account the value $m_{2}^{0}>\frac{1}{2}$ :

$$
\begin{gathered}
\lim _{\beta_{i} \rightarrow+\infty} \Delta^{\prime}=\lim _{\delta_{i} \rightarrow+0} \Delta^{\prime}= \\
=\pi^{-3 / 2} \int_{R^{3}} d p \rho_{01} e^{-p^{2}} \sup _{(t, x) \in R^{4}}\left|\frac{\partial \psi_{1}}{\partial t}+\left(\widehat{V}_{1}, \frac{\partial \psi_{1}}{\partial x}\right)+2 q_{1} \psi_{1}\left(p,\left[\bar{\omega}_{01}, \widehat{V}_{1}\right]\right)\right|+ \\
+\pi^{-3 / 2} \int_{R^{3}} d p \rho_{02} e^{-p^{2}} \sup _{(t, x) \in R^{4}}\left|\frac{\partial \psi_{2}}{\partial t}+\left(\widehat{V}_{2}, \frac{\partial \psi_{2}}{\partial x}\right)\right|+ \\
+\frac{4 d^{2} \rho_{01} \rho_{02}}{\pi^{2}} \sup _{(t, x) \in R^{4}} \psi_{1} \psi_{2} \int_{R^{3}} \int_{R^{3}} d u d u_{1} e^{-u^{2}-u_{1}^{2}}\left|\widehat{V}_{1}-\widehat{V}_{2}\right| .
\end{gathered}
$$

To obtain the statement of the theorem (46), it remains to make several transformations and use the integral (39). Thus, the theorem is proved.

Let us formulate a sufficient condition of infinitesimality of uniform-integral deviation for the approximate solution determined by Theorem 2 .

Corollary 3. Let the condition (44) be fulfilled for the first flow, that is, for $i=1$ or vectors $\bar{\omega}_{01}, \widehat{V}_{1}$ are parallel. The functions $\psi_{i}(t, x)$ have form (40) or (45). Then the error $\Delta$ will be arbitrarily small if additionally one of the conditions (41), (42) або (43) is valid. 
Since expression (46) differs from the limit $\Delta^{\prime}$ in Theorem 1, by adding

$$
\frac{4 q_{1} \rho_{01}}{\sqrt{\pi}}\left|\left[\bar{\omega}_{01}, \widehat{V}_{1}\right]\right| \sup _{(t, x) \in R^{4}} \psi_{1},
$$

then it is necessary to check that it vanishes. He goes to zero thanks to $q_{1} \rightarrow 0$ or $\left|\left[\bar{\omega}_{01}, \widehat{V}_{1}\right]\right|=$ 0 , because $\bar{\omega}_{01} \| \widehat{V}_{1}$.

Theorem 3. Assume that there are the relationships (14), (15) and functions (16) are bounded on $R^{4}$. Let degrees $m_{i}$ take the following values

$$
m_{1}=\frac{1}{4}, \quad m_{2}=m_{2}^{0} \in\left(\frac{1}{2} ;+\infty\right)
$$

and $\bar{\omega}_{01} \| \widehat{V}_{1}$.

Then, as before, there exists such a value $\Delta^{\prime}$, that the estimation (17) holds true, and the following statement is true

$$
\lim _{\beta_{i} \rightarrow+\infty} \Delta^{\prime}=\boldsymbol{Y}+\frac{4 q_{1}^{2} \rho_{01}}{\sqrt{\pi}}\left|\bar{\omega}_{01}\right|^{2} \sup _{(t, x) \in R^{4}}|x| \psi_{1} .
$$

Proof. At first we will get an expression for the value $\Delta^{\prime}$, in case of values of degrees (47), using a common form (37)

$$
\begin{gathered}
\Delta^{\prime}=\pi^{-3 / 2} \int_{R^{3}} d p \rho_{01} e^{-p^{2}} \sup _{(t, x) \in R^{4}} \mid \frac{\partial \psi_{1}}{\partial t}+\left(p \sqrt{\delta_{1}}+\widehat{V}_{1}+\left[\bar{\omega}_{01}, x\right] q_{1} \sqrt[4]{\delta_{1}}, \frac{\partial \psi_{1}}{\partial x}\right)- \\
-2 \psi_{1}\left(\left[\bar{\omega}_{01}, p\right],\left[\bar{\omega}_{01}, x\right]\right) q_{1}^{2} \mid+ \\
+\pi^{-3 / 2} \int_{R^{3}} d p \rho_{02} e^{-p^{2}} \sup _{(t, x) \in R^{4}} \mid \frac{\partial \psi_{2}}{\partial t}+\left(p \sqrt{\delta_{2}}+\widehat{V}_{2}+\left[\bar{\omega}_{02}, x\right] q_{2} \delta_{i}^{m_{2}^{0}}, \frac{\partial \psi_{2}}{\partial x}\right)+ \\
+2 \psi_{2}\left\{\left(p,\left[\bar{\omega}_{02}, \widehat{V}_{2}\right]\right) q_{2} \delta_{2}^{m_{2}^{0}-\frac{1}{2}}-\left(\left[\bar{\omega}_{02}, p\right],\left[\bar{\omega}_{02}, x\right]\right) q_{2}^{2} \delta_{2}^{2 m_{2}^{0}-\frac{1}{2}}\right\} \mid+ \\
+\frac{4 d^{2} \rho_{01} \rho_{02}}{\pi^{2}} \sup _{(t, x) \in R^{4}} \psi_{1} \psi_{2} \int_{R^{3}} \int_{R^{3}} d u d u_{1} e^{-u^{2}-u_{1}^{2}} \times \\
\times\left|\widehat{V}_{1}-\widehat{V}_{2}+u \sqrt{\delta_{1}}-u_{1} \sqrt{\delta_{2}}-\left[\bar{\omega}_{01} q_{1} \sqrt[4]{\delta_{1}}-\bar{\omega}_{02} q_{2} \delta_{2}^{m_{2}^{0}}, x\right]\right| .
\end{gathered}
$$

Next, let's pass to the low-temperature limit

$$
\begin{gathered}
\lim _{\beta_{i} \rightarrow+\infty} \Delta^{\prime}=\lim _{\delta_{i} \rightarrow+0} \Delta^{\prime}= \\
=\pi^{-3 / 2} \rho_{01} \int_{R^{3}} d p e^{-p^{2}} \sup _{(t, x) \in R^{4}}\left|\frac{\partial \psi_{1}}{\partial t}+\left(\widehat{V}_{1}, \frac{\partial \psi_{1}}{\partial x}\right)-2 \psi_{1}\left(\left[\bar{\omega}_{01}, p\right],\left[\bar{\omega}_{01}, x\right]\right) q_{1}^{2}\right|+ \\
+\pi^{-3 / 2} \rho_{02} \sup _{(t, x) \in R^{4}}\left|\frac{\partial \psi_{2}}{\partial t}+\left(\widehat{V}_{2}, \frac{\partial \psi_{2}}{\partial x}\right)\right| \int_{R^{3}} d p e^{-p^{2}}+ \\
+\frac{4 d^{2} \rho_{01} \rho_{02}}{\pi^{2}}\left|\widehat{V}_{1}-\widehat{V}_{2}\right| \int_{R^{3}} \int_{R^{3}} d u d u_{1} e^{-u^{2}-u_{1}^{2}} \sup _{(t, x) \in R^{4}} \psi_{1} \psi_{2},
\end{gathered}
$$

it remains to make some elementary transformations and calculate integrals that are included in the terms and we will get the limit (48). 
Remark 1. For infinitesimality of the error $\Delta$ according to the conditions of Theorem 3 , we can use consequence 3 , only removing from it the assumption $\bar{\omega}_{01}|| \widehat{V}_{1}$.

Theorem 4. Let the conditions (14), (15) and the boundness of all functions (16) on the time space $t$ and space coordinate $x$ remain true. The degrees $m_{i}$ have the values

$$
m_{1}=\frac{1}{4}, \quad m_{2}=\frac{1}{2}
$$

and $\bar{\omega}_{01} \| \widehat{V}_{1}$.

Then again there exists the value $\Delta^{\prime}$, inequality is being performed (17) and there is the equality

$$
\begin{gathered}
\lim _{\beta_{i} \rightarrow+\infty} \Delta^{\prime}= \\
=\boldsymbol{Y}+\frac{4}{\sqrt{\pi}}\left(q_{1}^{2} \rho_{01}\left|\bar{\omega}_{01}\right|^{2} \sup _{(t, x) \in R^{4}}\left(|x| \psi_{1}\right)+q_{2} \rho_{02}\left|\left[\bar{\omega}_{02}, \widehat{V}_{2}\right]\right| \sup _{(t, x) \in R^{4}} \psi_{2}\right) .
\end{gathered}
$$

Proof. Let's write a form of $\Delta^{\prime}$ in the case $m_{i}$ with values (49), using the general form (37)

$$
\begin{aligned}
\Delta^{\prime}=\pi^{-3 / 2} \rho_{01} \int_{R^{3}} d p e^{-p^{2}} \sup _{(t, x) \in R^{4}} \mid \frac{\partial \psi_{1}}{\partial t}+\left(p \sqrt{\delta_{1}}+\widehat{V}_{1}+\left[\bar{\omega}_{01}, x\right] q_{1} \sqrt[4]{\delta_{1}}, \frac{\partial \psi_{1}}{\partial x}\right)+ \\
+2 \psi_{1}\left\{\left(p,\left[\bar{\omega}_{01}, \widehat{V}_{1}\right]\right) \frac{q_{1}}{\sqrt{\delta_{1}}}-\left(\left[\bar{\omega}_{01}, p\right],\left[\bar{\omega}_{01}, x\right]\right) q_{1}^{2}\right\} \mid+ \\
+\pi^{-3 / 2} \rho_{02} \int_{R^{3}} d p e^{-p^{2}} \sup _{(t, x) \in R^{4}} \mid \frac{\partial \psi_{2}}{\partial t}+\left(p \sqrt{\delta_{2}}+\widehat{V}_{2}+\left[\bar{\omega}_{02}, x\right] q_{2} \sqrt{\delta_{2}}, \frac{\partial \psi_{2}}{\partial x}\right)+ \\
+2 \psi_{2}\left\{\left(p,\left[\bar{\omega}_{02}, \widehat{V}_{2}\right]\right) q_{2}-\left(\left[\bar{\omega}_{02}, p\right],\left[\bar{\omega}_{02}, x\right]\right) q_{2}^{2} \sqrt{\delta_{2}}\right\} \mid+ \\
\quad+\frac{4 d^{2} \rho_{01} \rho_{02}}{\pi^{2}} \sup _{(t, x) \in R^{4}} \psi_{1} \psi_{2} \int_{R^{3}} \int_{R^{3}} d u d u_{1} e^{-u^{2}-u_{1}^{2} \times} \\
\times\left|\widehat{V}_{1}-\widehat{V}_{2}+u \sqrt{\delta_{1}}-u_{1} \sqrt{\delta_{2}}-\left[\bar{\omega}_{01} q_{1} \sqrt[4]{\delta_{1}}-\bar{\omega}_{02} q_{2} \sqrt{\delta_{2}}, x\right]\right| .
\end{aligned}
$$

Taking into account the assumption of the theorem $\bar{\omega}_{01} \| \widehat{V}_{1}$, the term $\Delta^{\prime}$ is continuous by the variable $\delta_{i}$, when $\delta_{i} \rightarrow+0$. Arguing the possibility of a boundary transition in the same way as in Theorem 1 , we have

$$
\begin{gathered}
\lim _{\beta_{i} \rightarrow+\infty} \Delta^{\prime}=\lim _{\delta_{i} \rightarrow+0} \Delta^{\prime}= \\
=\pi^{-3 / 2} \rho_{01} \int_{R^{3}} d p e^{-p^{2}} \sup _{(t, x) \in R^{4}}\left|\frac{\partial \psi_{1}}{\partial t}+\left(\widehat{V}_{1}, \frac{\partial \psi_{1}}{\partial x}\right)-2 \psi_{1}\left(\left[\bar{\omega}_{01}, p\right],\left[\bar{\omega}_{01}, x\right]\right) q_{1}^{2}\right|+ \\
+\pi^{-3 / 2} \rho_{02} \sup _{(t, x) \in R^{4}}\left|\frac{\partial \psi_{2}}{\partial t}+\left(\widehat{V}_{2}, \frac{\partial \psi_{2}}{\partial x}\right)+2 \psi_{2}\left(p,\left[\bar{\omega}_{02}, \widehat{V}_{2}\right]\right)\right| \int_{R^{3}} d p e^{-p^{2}}+ \\
+\frac{4 d^{2} \rho_{01} \rho_{02}}{\pi^{2}}\left|\widehat{V}_{1}-\widehat{V}_{2}\right| \int_{R^{3}} \int_{R^{3}} d u d u_{1} e^{-u^{2}-u_{1}^{2}} \sup _{(t, x) \in R^{4}} \psi_{1} \psi_{2},
\end{gathered}
$$

applying the inequality of the triangle, the properties of the scalar and vector products, and, calculating the integrals, we obtain the assertion of the theorem (50). 
Corollary 4. Let the functions $\psi_{i}(t, x)$ have the form (40) or (45) and at least one of the conditions (41)-(43) is fulfilled. In addition, $q_{1} \rightarrow 0$, and there is one of the requirements:

$$
q_{2} \rightarrow 0
$$

or

$$
\bar{\omega}_{02} \| \widehat{V}_{2}
$$

Then the error $\Delta$ between the parts of the Bryan-Pidduck equation can be done arbitrarily small.

The conditions $q_{i} \rightarrow 0$ or $\bar{\omega}_{02} \| \widehat{V}_{2}$ vanish term

$$
q_{1}^{2} \rho_{01}\left|\bar{\omega}_{01}\right|^{2} \sup _{(t, x) \in R^{4}}\left(|x| \psi_{1}\right)+q_{2} \rho_{02}\left|\left[\bar{\omega}_{02}, \widehat{V}_{2}\right]\right| \sup _{(t, x) \in R^{4}} \psi_{2}
$$

functions $\psi_{i}$ from corollaries 1 and 2 with conditions (41)-(43) turn to zero the value $\mathbf{Y}$.

Now refuse the condition (14) for coefficient functions $\varphi_{i}$ and we will obtain several new approximate solutions of the equation (1).

Theorem 5. Let the condition (15) be fulfilled, and $\bar{\omega}_{0 i}|| \widehat{V}_{i}$. We will assume that the following expressions

$$
\varphi_{i} e^{\beta_{i} \bar{\omega}_{i}^{2} r_{i}^{2}},|x| \varphi_{i} e^{\beta_{i} \bar{\omega}_{i}^{2} r_{i}^{2}},\left|\frac{\partial \varphi_{i}}{\partial t}\right| e^{\beta_{i} \bar{\omega}_{i}^{2} r_{i}^{2}},\left|\frac{\partial \varphi_{i}}{\partial x}\right| e^{\beta_{i} \bar{\omega}_{i}^{2} r_{i}^{2}},|x|\left|\frac{\partial \varphi_{i}}{\partial x}\right| e^{\beta_{i} \bar{\omega}_{i}^{2} r_{i}^{2}}
$$

are bounded at the whole space $R^{4}$.

Then the value $\Delta$ is correctly determined and there exists such a value $\Delta^{\prime}$, that the estimation (17) is true, and depending on the values $m_{i}$ there is a statement

a) if $m_{i}>\frac{1}{2}$, then

$$
\lim _{\beta_{i} \rightarrow+\infty} \Delta^{\prime}=\sum_{i=1}^{2} \rho_{0 i} \sup _{(t, x) \in R^{4}}\left|\frac{\partial \varphi_{i}}{\partial t}+\left(\widehat{V}_{i}, \frac{\partial \varphi_{i}}{\partial x}\right)\right|+4 \pi d^{2} \rho_{01} \rho_{02}\left|\widehat{V}_{1}-\widehat{V}_{2}\right| \sup _{(t, x) \in R^{4}}\left(\varphi_{1} \varphi_{2}\right) ;
$$

b) in the case, when

$$
m_{1}=\frac{1}{2}, m_{2}=m_{2}^{0} \in\left(\frac{1}{2} ;+\infty\right)
$$

the equality is fulfilled

$$
\begin{gathered}
\lim _{\beta_{i} \rightarrow+\infty} \Delta^{\prime}=\rho_{01} \sup _{(t, x) \in R^{4}}\left|\frac{\partial \varphi_{1}}{\partial t}+\left(\widehat{V}_{1}, \frac{\partial \varphi_{1}}{\partial x}\right)\right| e^{q_{1}^{2}\left[\bar{\omega}_{01}, x\right]^{2}}+ \\
+\rho_{02} \sup _{(t, x) \in R^{4}}\left|\frac{\partial \varphi_{2}}{\partial t}+\left(\widehat{V}_{2}, \frac{\partial \varphi_{2}}{\partial x}\right)\right|+4 \pi d^{2} \rho_{01} \rho_{02}\left|\widehat{V}_{1}-\widehat{V}_{2}\right| \sup _{(t, x) \in R^{4}}\left(\varphi_{1} \varphi_{2} e^{q_{1}^{2}\left[\bar{\omega}_{01}, x\right]^{2}}\right) ;
\end{gathered}
$$

c) and, finally, if $m_{i}=\frac{1}{2}$, then

$$
\begin{aligned}
& \lim _{\beta_{i} \rightarrow+\infty} \Delta^{\prime}=\sum_{i=1}^{2} \rho_{0 i} \sup _{(t, x) \in R^{4}} e^{q_{i}^{2}\left[\bar{\omega}_{0 i}, x\right]^{2}}\left|\frac{\partial \varphi_{i}}{\partial t}+\left(\widehat{V}_{i}, \frac{\partial \varphi_{i}}{\partial x}\right)\right|+ \\
& +4 \pi d^{2} \rho_{01} \rho_{02}\left|\widehat{V}_{1}-\widehat{V}_{2}\right| \sup _{(t, x) \in R^{4}}\left(\varphi_{1} \varphi_{2} e^{q_{1}^{2}\left[\bar{\omega}_{01}, x\right]^{2}+q_{2}^{2}\left[\bar{\omega}_{02}, x\right]^{2}}\right) .
\end{aligned}
$$


Proof. To begin with, using an estimation (28), the Maxwellian form $M_{i}(11)$ and taking into account the equality (27) we obtain

$$
\begin{gathered}
\int_{R^{3}} d V \int_{R^{3}} d \omega|D(f)-Q(f, f)| \leqslant \\
\leqslant I^{3 / 2} \pi^{-3} \sum_{i=1}^{2} \rho_{0 i} \beta_{i}^{3} \int_{R^{3}} d V \int_{R^{3}} d \omega e^{\beta_{i} \bar{\omega}_{i}^{2} r_{i}^{2}}\left|\frac{\partial \varphi_{i}}{\partial t}+\left(\widehat{V}_{i}, \frac{\partial \varphi_{i}}{\partial x}\right)\right| e^{-\beta_{i}\left(\left(V-\bar{V}_{i}\right)^{2}+I \omega^{2}\right)}+ \\
+\frac{4 d^{2} \rho_{01} \rho_{02}}{\pi^{2}} e^{\beta_{1} \bar{\omega}_{1}^{2} r_{1}^{2}+\beta_{2} \bar{\omega}_{2}^{2} r_{2}^{2}} \varphi_{1} \varphi_{2} \int_{R^{3}} \int_{R^{3}} d u d u_{1} e^{-u^{2}-u_{1}^{2}}\left|\widehat{V}_{1}-\widehat{V}_{2}+\frac{u}{\sqrt{\beta_{1}}}-\frac{u_{1}}{\sqrt{\beta_{2}}}-\left[\bar{\omega}_{1}-\bar{\omega}_{2}, x\right]\right| .
\end{gathered}
$$

Calculating the integral on $\omega$, and performing a replacement (32) we have the following estimation

$$
\begin{gathered}
\int_{R^{3}} d V \int_{R^{3}} d \omega|D(f)-Q(f, f)| \leqslant \\
\leqslant \pi^{-3 / 2} \sum_{i=1}^{2} \rho_{0 i} \int_{R^{3}} d p e^{\beta_{i} \bar{\omega}_{i}^{2} r_{i}^{2}}\left|\frac{\partial \varphi_{i}}{\partial t}+\left(\widehat{V}_{i}+\left[\bar{\omega}_{i}, x\right]+\frac{p}{\sqrt{\beta_{i}}}, \frac{\partial \varphi_{i}}{\partial x}\right)\right| e^{-p^{2}}+ \\
+\frac{4 d^{2} \rho_{01} \rho_{02}}{\pi^{2}} e^{\beta_{1} \bar{\omega}_{1}^{2} r_{1}^{2}+\beta_{2} \bar{\omega}_{2}^{2} r_{2}^{2}} \varphi_{1} \varphi_{2} \int_{R^{3}} \int_{R^{3}} d u d u_{1} e^{-u^{2}-u_{1}^{2}}\left|\widehat{V}_{1}-\widehat{V}_{2}+\frac{u}{\sqrt{\beta_{1}}}-\frac{u_{1}}{\sqrt{\beta_{2}}}-\left[\bar{\omega}_{1}-\bar{\omega}_{2}, x\right]\right| .
\end{gathered}
$$

Passing to the supremum in the inequality by all possible values $(t, x)$, the existence of which ensures the condition of the theorem (51), we will have an expression for the value $\Delta^{\prime}$ :

$$
\begin{aligned}
\Delta \leqslant \Delta^{\prime}= & \pi^{-3 / 2} \sum_{i=1}^{2} \rho_{0 i} \int_{R^{3}} d p \sup _{(t, x) \in R^{4}} e^{\beta_{i} \bar{\omega}_{i}^{2} r_{i}^{2}}\left|\frac{\partial \varphi_{i}}{\partial t}+\left(\widehat{V}_{i}+\left[\bar{\omega}_{i}, x\right]+\frac{p}{\sqrt{\beta}}, \frac{\partial \varphi_{i}}{\partial x}\right)\right| e^{-p^{2}}+ \\
& +\frac{4 d^{2} \rho_{01} \rho_{02}}{\pi^{2}} \sup _{(t, x) \in R^{4}} e^{\beta_{1} \bar{\omega}_{1}^{2} r_{1}^{2}+\beta_{2} \bar{\omega}_{2}^{2} r_{2}^{2}} \varphi_{1} \varphi_{2} \times \\
& \times \int_{R^{3}} \int_{R^{3}} d u d u_{1} e^{-u^{2}-u_{1}^{2}}\left|\widehat{V}_{1}-\widehat{V}_{2}+\frac{u}{\sqrt{\beta_{1}}}-\frac{u_{1}}{\sqrt{\beta_{2}}}-\left[\bar{\omega}_{1}-\bar{\omega}_{2}, x\right]\right| .
\end{aligned}
$$

Similarly, as in Theorem 1, we perform a re-termination $\delta_{i}=\frac{1}{\beta_{i}}$ and substitute an expression (15) for the angular velocity of the flow $\bar{\omega}_{i}$, using the condition $\bar{\omega}_{0 i}|| \widehat{V}_{i}$ :

$$
\begin{gathered}
\Delta^{\prime}=\pi^{-3 / 2} \sum_{i=1}^{2} \rho_{0 i} \int_{R^{3}} d p e^{-p^{2}} \sup _{(t, x) \in R^{4}} e^{\delta_{i}^{2 m_{i}-1} q_{i}^{2}\left[\bar{\omega}_{0 i}, x\right]^{2}} \times \\
\times\left|\frac{\partial \varphi_{i}}{\partial t}+\left(p \sqrt{\delta}+\widehat{V}_{i}+\delta_{i}^{m_{i}} q_{i}\left[\bar{\omega}_{0 i}, x\right], \frac{\partial \varphi_{i}}{\partial x}\right)\right|+ \\
+\frac{4 d^{2} \rho_{01} \rho_{02}}{\pi^{2}} \sup _{(t, x) \in R^{4}} \varphi_{1} \varphi_{2} e^{\delta_{1}^{2 m_{1}-1} q_{1}^{2}\left[\bar{\omega}_{01}, x\right]^{2}+\delta_{2}^{2 m_{2}-1} q_{2}^{2}\left[\bar{\omega}_{02}, x\right]^{2}} \int_{R^{3}} \int_{R^{3}} d u d u_{1} e^{-u^{2}-u_{1}^{2}} \times \\
\times\left|\widehat{V}_{1}-\widehat{V}_{2}+u \sqrt{\delta_{1}}-u_{1} \sqrt{\delta_{2}}-\left[\bar{\omega}_{01} q_{1} \delta_{1}^{m_{1}}-\bar{\omega}_{02} q_{2} \delta_{2}^{m_{2}}, x\right]\right| .
\end{gathered}
$$

Since the theorem is considered with $m_{i} \geqslant \frac{1}{2}$, then expression (55) is continuous by the variable $\delta_{i}$, when $\delta_{i} \rightarrow+0$ and, using the continuity lemma of supremum [6], one can go to the limit in the last equality. Performing the limiting passage when $\delta_{i} \rightarrow+0$, what is responsible to $\beta_{i} \rightarrow+\infty$, depending on the values of $m_{i}$ and after the calculation of the integrals, we obtain the limits (52), (5) and (54). 
Corollary 5. Let the functions $\varphi_{i}$ have the same form as the functions $\psi_{i}$, i.e. (40) and (45) and satisfy the conditions of Theorem 5. Assume also that is executed at least one of the conditions (41)-(43) takes place. Then the deviation (8) can be made arbitrarily small.

Direct substitution of functions $\varphi_{i}$ of the form (40) or (45) will do equal to zero the terms under the supremum sign in the expressions (52), (5) and (54), and the validity of any of the conditions (41) - (43) will avoid the last term in the terms under consideration.

On the class of obtained solutions, we can note that these are smooth functions, as follows from the conditions of the theorems and consequences in the work. We do not consider other properties of the solutions obtained in this paper, since our aim is to obtain the explicit form of some new approximate solutions of the Bryan-Pidduck equation.

4. Conclusions. In the work, the new approximate solutions of the Boltzmann equation for model of rough spheres are obtained. In this model, in addition to the translational motion of the molecule, also rotation presents, which makes it more interesting to study. New solutions have a bimodal distribution structure, namely linear combination of exact solutions, Maxwellians, with coefficient functions depends on time and spatial coordinates. As exact solutions the so-called screws, heterogeneous Maxwellians are considered describing flow of gas revolving around some fixed axis. Physically the resulting solutions can be interpreted as follows: when combined two rotating flows are the slowdown of rotation of generated stream. It is worth noting that for a model of rough spheres the asymmetric flows are considered in this work for the first time, that is, those that have different levels of rotation, which allows some others to get approximation of solutions for the kinetic Bryan-Pidduck equation. Infinitesimality of a uniform-integral deviation between parts of the studied equation is achieved by imposing some conditions on hydrodynamic parameters of flows, such as a translational part of mass velocity, a vector of rotation of the flow, a diameter of the gas particle.

\section{REFERENCES}

1. S. Chapman, T.G. Cowling, The mathematical theory of non-uniform gases, Cambridge Univ. Press, Cambridge, 1952.

2. G.H. Bryan, On the application of the determinantal relation to the kinetic theory of polyatomic gases, Rept. Brit. Assoc. Adv. Sci., 64 (1894), 102-106.

3. F.B. Pidduck, The kinetic theory of a special type of rigid molecule, Proc. Roy. Soc., A101 (1922), 101-110.

4. C. Cercignani, M. Lampis, On the kinetic theory of a dense gas of rough spheres, J. Statist. Phys., 53 (1988), 655-672.

5. V.D. Gordevskyy, Explicit approximate solutions of the Boltzmann equation for the model of rough spheres, Reports of the National Academy of Sciences of Ukraine, 4 (2000), 10-13. (in Ukrainian)

6. V.D. Gordevskyy, Approximate billow solutions of the kinetic Bryan-Pidduck equation, Math. Meth. Appl. Sci., 23 (2000), 1121-1137.

7. V.D. Gordevskii, A.A. Gukalov Interaction of locally Maxwellian flows in the model of rough spheres, Theor. Math. Phys, 176 (2013), №2, 322-336. (in Russian)

8. V.D. Gordevskyy, A.A. Gukalov, Interaction of the eddy flows in the Bryan-Pidduck model, Vysnik Kharkiv Univ., Ser. Mat. Prykl. Mat. Mech., 64 (2011), №2, 27-41. (in Russian)

9. V.D. Gordevskyy, A.A. Gukalov, Maxwell distributions in a model of rough spheres, Ukr. Mat. Zh., 63 (2011), №5, 629-639. (in Russian)

10. V.D. Gordevskyy, E.S. Sazonova Asymmetrical bimodal distributions with screw modes, Math. Phys., Anal., Geom., 3 (2011), V.7, 212-224. 
11. C. Cercignani, Theory and Application of the Boltzmann Equation, Elsevier, New York, 1975.

V.N. Karazin Kharkiv National University, Kharkiv, Ukraine gordevskyy2006@gmail.com

B.Verkin Institute for Low Temperature Physics and Engineering, Kharkiv, Ukraine hukalov@ilt.kharkov.ua

Received? 